\section{FOEP: A FORTRAN-based experimental package}

\author{
ROBERT B. ALLEN \\ University of California, San Diego, La Jolla, California 92093
}

Reaction-time (RT) experiments may require the presentation of sets of questions in which all but one of the words are the same from trial to trial. For example, in a study of impression formation, Allen, Ebbesen, and Bessman (Note 1) asked, "Was he sincere?" and "Was he generous?" Alternatively, experiments may require a pairwise (factorial) presentation of segments within a constant framework; for example, "Are all generous people friendly?" or "Are all kind people cold?" (Ebbesen \& Allen, in press). Finally, questions may be so distinct that no part of the questions is constant, yet the order of the questions must be randomized. In adition to binary forced-choice responses, RT procedures frequently require confidence ratings. FOEP (FORTRAN Experimental Package) provides a system which can easily accommodate these and many other experimental requirements. Despite the flexibility of the system, it is designed to guide the user easily to a satisfactory experimental configuration.

During initialization of a stimulus file, the system interrogates the user about three general aspects of the experimental design. First, the user is asked about the parameters of the design: the number of fixed (consistent from question to question) segments, whether the stimuli are to be randomized, and whether a one- or twodimensional presentation of the variable segment is required. In the second phase, a calling sequence is requested. The calling sequence is a string of coded numbers that represents the temporal order with which events are to be presented to the participant on each trial. For instance, the user might first request that a READY signal be displayed for $.5 \mathrm{sec}$, then that the screen be erased and the experimental question be presented to the participant until a response is made. In the third phase, the user is asked to enter the fixed and variable segments which compose the questions and other verbal stimuli (e.g., READY) that are to be presented to the participants. Once a stimulus file has been created, it is saved and then reinstated when data are to be collected. The participants' data are stored in files for later examination by FOEP or for direct analysis by specifically designed programs.

FOEP allows a variety of factorializations of the variable segments. Elements of a list of segments may be combined either with elements of the same list or with elements of another list. In the former case, there are options for the inclusion of the diagonal and/or the presentation of only one half of the factorial design.

The development of this system was supported in part by NIMH Grant MH 26069 to Ebbe B. Ebbesen.
When the elements of two different lists are presented, the lists may be the same length or different lengths.

In the usual operating procedure, three data points may be collected per trial. These are (1) the RT, (2) the code of the response made to RT judgment, and (3) a numeric rating. In order to assess the stability of responses, blocked replications of the questions may be presented.

Program Limitations. At present, 60 characters are permitted in each segment, and a total of 30 fixed and variable segments are allowed in each stimulus file. For factorial designs, the latter restriction is generally not a problem. For experiments in which each question is unique (i.e., in which the question takes one complete segment), stimulus file space can be a problem. Therefore, FOEP provides the facility to chain automatically from one stimulus file to another. Finally, FOEP allows for the collection of a maximum of 200 data triplets (RT, response, and rating) for any one stimulus file replication. Factorial designs larger than 14 by 14 are not allowed.

A FORTRAN-based system may not completely optimize the efficiency of the system, but a high-level language facilitates alterations of the system by many users who are not familiar with the computer's assembly code. For example, modification of each of the limitations described above could be made with changes to only a few lines in the system program.

Program Requirements. As presently configured, FOEP requires approximately $14 \mathrm{~K}$ of core in a PDP $8 / \mathrm{A}$ or $8 / E$. Modifications of either the stimulus or data file size or separation of the system into independently called programs can substantially reduce the core requirement. FOEP is written in Digital FORTRAN II to run under the OS/8 operating system. FOEP could be easily adapted to other operating systems that have a FORTRAN compiler if patches between the FORTRAN code and the system assembly code are easily made. The patches are required so that clock ticks may be used to calculate RTs and timed delays. At present, FOEP is operated with a digital VT50 video terminal, a RX8 dual floppy disk system, and a DK8-ES real-time clock. Presumably, any high-storage terminal can be substituted for the VT50. DECtape or cassette mass storage can be substituted for the floppy disks. And standard "line clocks" may be used to replace the DK8-ES, although this will generally decrease RT accuracy. Responses of participants have usually been made by keypresses on the VT50 keyboard, but a direct I/O interface could be easily developed.

Availability. Printed copies of a source listing, as well as a user's manual which includes sample programs, may be obtained without charge from Robert B. Allen, Department of Psychology C-009, University of California, San Diego, La Jolla, California 92093. 


\section{REFERENCE NOTE}

1. Allen, R. B., Ebbesen, E. B., \& Bessman, E. Cognitive processes in person perception: Retrieval of trait and behavioral information. Manuscript, University of California, San Diego, 1977.

\section{REFERENCE}

Ebbesen, E. B., \& Allen, R. B. Cognitive processes in implicit personality inferences. Journal of Personality and Social Psychology, in press.

(Accepted for publication October 6, 1977.) 